\title{
Performance Comparison of Scheduling Algorithms for Peer-to-Peer Collaborative File Distribution
}

\author{
Jonathan S. K. Chan, Student Member, IEEE, Victor O. K. Li, Fellow, IEEE, and King-Shan Lui, Member, IEEE
}

\begin{abstract}
Peer-to-Peer file sharing applications in the Internet, such as BitTorrent, Gnutella, etc., have been immensely popular. Prior research mainly focuses on peer and content discovery, overlay topology formation, fairness and incentive issues, etc. However, little attention has been paid to investigate the data distribution problem which is also a core component of any file sharing application. In this paper, we present the first effort in addressing this collaborative file distribution problem and formally define the scheduling problem in a simplified context. We develop several algorithms to solve the problem and study their performance. We deduce a theoretical bound on the minimum download time experienced by users and also perform simulations to evaluate our algorithms. Simulation results show that our graph-based dynamically weighted maximum-flow algorithm outperforms all other algorithms. Therefore, we believe our algorithm is a promising solution to be employed as the core scheduling module in P2P file sharing applications.
\end{abstract}

Index Terms - Peer-to-Peer, P2P, file sharing, data distribution, scheduling algorithms.

\section{INTRODUCTION}

$\mathbf{P}$ EER-TO-PEER (P2P) applications have become immensely popular in the Internet. One of the most popular applications of $\mathrm{P} 2 \mathrm{P}$ networks is collaborative sharing of large video/audio files and software. Traditional methods for file sharing, such as the client/server approach (e.g. FTP, WWW), suffer from scalability bottleneck. As the outgoing bandwidth of the server is shared among all simultaneous clients, the more the clients, the less bandwidth each client can have. Hence, the performance of client/server approach deteriorates rapidly as the number of simultaneous clients increases. P2P file sharing solves the problem by allowing peers to act as servers. Interestingly, in a well-designed P2P file sharing network, more peers participating in the file sharing session generally means better performance, as each peer could download simultaneously from multiple peers. Due to the significant performance improvements with collaborative file sharing, there has been widespread use of P2P file sharing applications like BitTorrent [1], Gnutella [2], Kazaa [3], Napster [4], etc. A recent study [5] shows that BitTorrent traffic accounts for an amazing $35 \%$ of all Internet traffic, which is more than any other single protocol, demonstrating the increasing importance of P2P file sharing systems among the Internet community.

Manuscript received December 14, 2005; revised July 12, 2006. This research is supported in part by the Areas of Excellence Scheme, established by the University Grants Committee, Hong Kong Special Administrative Region, China, Project No. AoE 99-01.

The authors are with the Department of Electrical and Electronic Engineering, University of Hong Kong, Pokfulam, Hong Kong, China. (e-mail: skjchan@eee.hku.hk, vli@eee.hku.hk, kslui@eee.hku.hk).

Digital Object Identifier 10.1109/JSAC.2007.070115.
Experiments in [6] have shown that parallel downloading scheme in P2P file sharing systems could result in higher aggregate download rate and thus shorter download time. In parallel downloading, an end user opens multiple connections with multiple file sources to download different portions of the file from different sources and then reassembles the file locally. According to the analysis in [7], the service capacity increases greatly compared with schemes that share the file as a whole.

Prior research in P2P file sharing usually focuses on topics like overlay topology formation, peer discovery, content search, sharing fairness, incentive mechanisms, etc. One of the core components of file sharing systems, the data distribution scheduling problem, on the other hand, has received little attention. The scheduling problem is important because it governs how file pieces are transmitted and distributed among peers, and has a direct effect on the performance. A poor data distribution schedule could result in considerably longer download time, while a good schedule could shorten the completion time and efficiently utilize all resources like network bandwidth. This article studies the "data distribution scheduling problem" in P2P collaborative file sharing systems and proposes a novel maximum flow graph model for efficiently solving the scheduling problem.

Our major contributions are summarized as follows:

- We formally define the data distribution scheduling problem using matrix formulations with granularity down to each file piece possessed by each peer. We also derive a theoretical lower bound of the transmission time required.

- We develop several algorithms (including a novel graphbased maximum-flow algorithm) for determining the file piece distribution schedule, and evaluate their performance by simulations.

- Simulation results show that our dynamically weighted maximum-flow algorithm outperforms other algorithms.

The rest of this paper is organized as follows. Section II briefly describes some related work. The communication model, notations and analysis are given in Section III. Section IV presents various algorithms for approaching the scheduling problem, followed by an evaluation using computer simulations in Section V. Some of our future research directions are given in Section VI and we conclude the paper in Section VII.

Due to space limitations, we have removed detailed explanations, figures, proofs and examples. We refer interested readers to [17] for the full version of this paper. 


\section{RELATED WORK}

Due to the dramatic increase of broadband user population, there has been large-scale deployment of P2P file sharing systems in the Internet. BitTorrent (BT) [1], [8] is perhaps the most representative one. Tracker servers maintain a list of participating peers. When a new peer joins, the tracker server will send the new peer the contact information of a few connected peers from the list. Then, this new peer contacts these peers directly for downloading the file. A shared file is partitioned into multiple small pieces, usually about 256 Kbytes or 512Kbytes in size. Peers exchange information on which pieces they currently possess, and request missing pieces from others. A peer can maximize its download speed by requesting different pieces from different peers at the same time. A scheduling mechanism is needed for a peer to decide which pieces to request and to whom such a request should be made. A poor scheduling algorithm may lead to every peer getting nearly the same set of pieces and consequently decreases the number of file piece sources which a peer can simultaneously download from. BT employs the Rarest Element First (REF) algorithm, in which those pieces that most peers do not have are downloaded first. This algorithm is good at increasing the availability of different file pieces in the network and is efficient in distributing all pieces from the original source to different peers across the network. However, our simulation results show that $R E F$ is not an optimal scheduling algorithm.

The self-scaling properties of collaborative file distribution architectures, where each peer has equal upload and download rates of $b$, have been shown in [9]. If the file size is $f$, the file is chopped into $m$ pieces, and let $\tau=\frac{f}{b}$ be the time needed for transmitting the whole file, it would take Time $=1+$ $\left\lfloor\log _{k} N\right\rfloor \frac{k \tau}{m}$ to serve all the $N$ peers organized in $k$ spanning trees. The time required increases only logarithmically with increasing number of peers $N$ in collaborative distribution, as opposed to a linear increase in the case of client-server distribution strategies.

Two crucial factors that affect the global effectiveness of the file distribution process are evaluated in [10]. They are "peer selection strategies" and "file piece selection strategies," and they have direct impacts on the delay experienced by the peers and the global throughput of the system. It is found that the most missing peer selection strategy, in which the peer with the largest number of un-received file pieces is selected for transmission first, can minimize the download time of the last complete peer and produce a smaller variance of the download times of the peers. This is because the most missing peer selection strategy ensures regular progress of all the peers by having an even dissemination of the file pieces. On the other hand, for file piece selection strategies, it is concluded that the rarest piece first strategy shows significant performance improvement over the random piece selection strategy. Some of our algorithms presented in Section IV are basically in the same spirit with the evaluations presented in [10] by employing the most missing peer selection and the rarest file piece selection strategies.

The problem of broadcasting or multicasting a single message in heterogeneous networks has been investigated in [11],
[12] from the algorithmic point of view. In particular, [11] studies the problem in a network where nodes have different processing times and the transmission times between different node pairs also vary. The authors evaluate the completion time of various algorithms and show that the well-known Fastest Node First algorithm may result in solutions which are worse than the optimal by an unbounded factor. They subsequently propose the Fastest Edge First and Earliest Completing Edge First algorithms to better solve the problem. Reference [12] studies the problem in a similar network as [11] except that all transmission times are assumed to be the same. It proves that the problem of minimizing the maximum completion time of broadcasting a single message is NP-hard. It also shows the Fastest Node First heuristic in computing broadcast schedules can produce an 1.5 approximation schedule for the same problem. It is worth noting that [11], [12] only analyze the problem of distributing one single message. In $\mathrm{P} 2 \mathrm{P}$ file sharing systems, a file is divided into multiple file pieces instead of one big piece. Therefore, efficient scheduling algorithms have to be developed and this is the aim of our work. To the best of our knowledge, this paper presents the first effort in addressing this problem based on some simplifications.

Some measurement-based research, such as those in [13], reveals the inherent difficulty of the peer selection problem and recognizes the selection of peers would have profound effect on the overall performance. They tried to use some bandwidth probing techniques to optimize the selection of "good" peers. On the other hand, some analytical models [7], [14] have been proposed. In [7], a branching process and a Markov chain model are proposed to study the transient regime and the steady state regime of the BitTorrent system, respectively. A fluid model is presented in [14], where the expressions of the numbers of incomplete peers and seeds (completed peers) could be obtained from the parameters such as the peer arrival rate, departure rate and the upload/download rates. However, previous models just consider the network, a particular peer or a particular file-sharing session as a whole, while our work is the first to model the data traffic transmission as a matrix representation down to the granularity of each file piece possessed by each peer. We also care about the scheduling selection of "good" peers, as well as "good" file pieces.

We have done a preliminary study in [15] with a homogeneous network assumption, where all the peers have symmetric upload and download bandwidths and the transmission times between any node pairs are the same. In this special case, we proposed a graph-based weighted Bipartite Matching (BPM) algorithm, which can efficiently and optimally solve all the cases tested. In this paper, we shall extend our results to networks with asymmetric bandwidth allocation and develop algorithms that can efficiently solve the problem.

\section{Problem Definition}

\section{A. Communication Model}

We assume users are situated at the edge of the network, with logical links connecting every pair of peers (i.e. fullyconnected graph, an example network is shown in Fig. 1).

1) Synchronous Scheduling: Due to the initial complexity we face when first studying this problem, we begin 


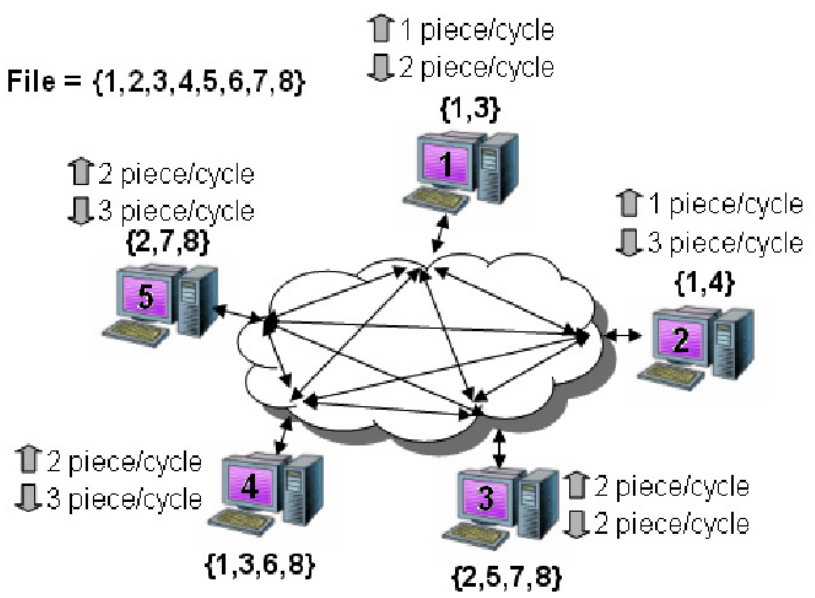

Fig. 1. Fully-connected network with asymmetric bandwidth allocation.

our investigation by assuming the transmission times between any node pairs are the same. That is, the data distribution scheduling is performed in discrete cycles synchronously.

2) Exchange of Information: As in BitTorrent, each peer actively communicates with other actively connected peers to announce his own set of possession information about which file pieces he currently possesses. This possession information is sent in the form of a bit-vector, say for example Peer 4 will announce $\{1,0,1,0,0,1,0,1\}$ to others, meaning that he currently possesses file pieces $1,3,6$, and 8 . For a typical video file of 650 Mbytes with each file piece having a size of $512 \mathrm{Kbytes}$, we will have 1300 bits ( 163bytes) in the bit-vector, which is small and will not consume too much bandwidth relative to the actual transmission of the data file piece itself. In the following sections in this paper, these possession bit-vectors are assumed to be exchanged without errors between all connected peers before the scheduling of the next cycle.

3) Asymmetric Bandwidth: Each peer may have asymmetric upload and download bandwidths. This is common in the Internet where most users connect to the network using the Asymmetric Digital Subscriber Line (ADSL) technology. We assume the nodes can only send or receive an integral number of file pieces for each cycle. An example is shown in Fig. 1, where Peer 1 can send out one file piece and receive two pieces from others for each cycle, and Peer 2 can send one file piece and receive three pieces for each cycle, etc.

4) Quasi-Static Assumption: In this paper, we assume all the peers will not leave the file sharing session once initiated until the distribution process completes. Although this assumption is unlikely to hold in real systems, where peers may come and go and the peers may switch to exchange with different sets of peers during the distribution process, we could reasonably extend the notion of this quasi-static assumption to represent a particular period, say one minute, during which the peers keep active exchanges with this particular set of peers and we aim at transmitting the largest amount of data during this period by employing our proposed scheduling algorithms.

5) Notations and Definitions: Let the number of participating peers be $N$ and the number of file pieces be $M$. The shared file $F$ is chopped into $M$ smaller pieces $F=\left\{F_{1}, F_{2}, \ldots, F_{M}\right\}$ and each peer possesses a subset of $F$. We represent the file piece possession information as an $N \times M$ matrix $P$, called the possession matrix. $P_{i j}=1$ if and only if node $i$ possesses file piece $F_{j}$ $(1 \leq i \leq N, 1 \leq j \leq M)$; otherwise $P_{i j}=0$. We use $P^{t}$ to denote the possession matrix at time $t$. Refer to Fig. 1 where $F=\left\{F_{1}, F_{2}, \ldots, F_{8}\right\}$ and $\{\ldots\}$ next to a node indicates the pieces that the node possesses, the possession matrix at the beginning $(t=0)$ is:

$$
P^{0}=\left(\begin{array}{llllllll}
1 & 0 & 1 & 0 & 0 & 0 & 0 & 0 \\
1 & 0 & 0 & 1 & 0 & 0 & 0 & 0 \\
0 & 1 & 0 & 0 & 1 & 0 & 1 & 1 \\
1 & 0 & 1 & 0 & 0 & 1 & 0 & 1 \\
0 & 1 & 0 & 0 & 0 & 0 & 1 & 1
\end{array}\right)
$$

Due to the synchronous scheduling assumption mentioned in Section III.A.1, the data distribution can be made in discrete cycles synchronously. Given an initial possession matrix $P^{0}$, after one cycle of file piece distribution, a new possession matrix $P^{1}$ will be formed. That is, $P^{k}$ denotes the possession matrix after $k$ cycles.

In each cycle, Peer $i$ can send out at most $p_{i}$ file pieces and receive at most $q_{i}$ file pieces from others. We use two integer vectors $p=\left\{p_{1}, p_{2}, \ldots, p_{N}\right\}$ and $q=\left\{q_{1}, q_{2}, \ldots, q_{N}\right\}$ to denote the upload and download limits for the peers. For example in Fig. $1, p=\{1,1,2,2,2\}$ and $q=\{2,3,2,3,3\}$. Note that it is usual to have $p_{i}$ smaller than $q_{i}$ in asymmetric bandwidth allocation schemes, such as ADSL. Nonetheless, our algorithms and analysis presented in later sections are not restricted by whether $p_{i}$ is smaller or larger than $q_{i}$.

We also refer to a possession matrix as a problem instance. A problem instance $P$ is feasible if for each file piece in $\left\{F_{1}, F_{2}, \ldots, F_{M}\right\}$, at least one peer possesses it, i.e. $\sum_{i=1}^{N} P_{i j} \geq$ $1, \forall j \in[1, M]$. A problem instance is infeasible if it is not feasible, meaning that there is no way for every peer to get all file pieces since there is at least one file piece not available in the system.

A schedule specifies how file pieces are distributed among peers. At each cycle, for each peer, it specifies which file pieces the peer has to send out and to whom. A possible schedule for $P^{0}$ above with $p=\{1,1,2,2,2\}$ and $q=$ $\{2,3,2,3,3\}$ is:

Node 1: send Piece 3 to Node 2

Node 2: send Piece 4 to Node 1

Node 3: send Piece 5 to Node 1, send Piece 5 to Node 2

Node 4: send Piece 6 to Node 2, send Piece 6 to Node 3

Node 5: send Piece 2 to Node 4, send Piece 7 to Node 4

Formally, we use an $N \times M$ matrix $S$ to represent the schedule in one cycle, which specifies which pieces a peer receives and from whom. $S_{i j}=x$ if and only if node $i$ receives file piece $j$ from node $x$, otherwise $S_{i j}=0$. From $S$, we can derive the piece transmission matrix $T$ (also $N \times M$ ), which ignores the sender identities and only specifies which 
pieces a peer receives. $T_{i j}=1$ if and only if $S_{i j} \neq 0$, otherwise $T_{i j}=0$. Similar to $P$, we use superscripts to refer to different cycles. That is, $S^{k}$ and $T^{k}$ are the schedule and piece transmission matrix at cycle $k$, respectively. For the above example schedule, we have:

$$
\begin{aligned}
S^{0} & =\left(\begin{array}{llllllll}
0 & 0 & 0 & 2 & 3 & 0 & 0 & 0 \\
0 & 0 & 1 & 0 & 3 & 4 & 0 & 0 \\
0 & 0 & 0 & 0 & 0 & 4 & 0 & 0 \\
0 & 5 & 0 & 0 & 0 & 0 & 5 & 0 \\
0 & 0 & 0 & 0 & 0 & 0 & 0 & 0
\end{array}\right) \\
T^{0} & =\left(\begin{array}{llllllll}
0 & 0 & 0 & 1 & 1 & 0 & 0 & 0 \\
0 & 0 & 1 & 0 & 1 & 1 & 0 & 0 \\
0 & 0 & 0 & 0 & 0 & 1 & 0 & 0 \\
0 & 1 & 0 & 0 & 0 & 0 & 1 & 0 \\
0 & 0 & 0 & 0 & 0 & 0 & 0 & 0
\end{array}\right)
\end{aligned}
$$

Node 1 receives Piece 4 sent from Node 2 and Piece 5 sent from Node 3. Therefore, $S_{14}^{0}=2, S_{15}^{0}=3$ and $T_{14}^{0}=1$, $T_{15}^{0}=1$.

There are several properties that a valid schedule (a schedule that does not violate any assumptions) should observe:

- At least one file piece must be distributed among the peers in each cycle (at least one entry in $S$ and $T$ is non-zero)

- A node cannot send a piece that it does not possess (if $S_{i j}=x$, then $P_{x j}=1$ )

- Node $x$ cannot send more than $p_{x}$ file pieces in a cycle (at most $p_{x} x$ 's in $S$ )

- Node $x$ cannot receive more than $q_{x}$ file pieces in a cycle (sum of row $x$ in $T$ is at most $q_{x}$ )

- Node $x$ cannot send a piece to Node $y$ that Node $y$ already has $\left(S_{y j}^{k}=0\right.$ and $T_{y j}^{k}=0$ if $P_{y j}^{k}=1$ for the same $y, j$ at any cycle $k$ )

Given the possession matrix $P^{k-1}$ and a valid schedule $S^{k-1}, T^{k-1}$ at cycle $k-1$, the possession matrix at cycle $k$ (for $k>0$ and $k$ is an integer) is given by,

$$
P^{k}=P^{k-1}+T^{k-1} \text {. }
$$

Intuitively, given an initial feasible $P^{0}$ and a sequence of valid schedules, after a certain, say $k_{0}$, cycles, $P_{i j}^{k_{0}}=1, \forall i, j$, all peers will get all the file pieces eventually and the file distribution can terminate. In other words, $k_{0}$ is the time needed for complete distribution of the whole file to all peers. An optimal schedule is a schedule that requires the minimum number of cycles for completion among all possible schedules. Our goal is to develop algorithms that aim at finding optimal schedules.

\section{B. Analysis}

In this section, we analyze the lower bound of $k_{0}$, which is the number of cycles needed for complete distribution of the whole file to all peers. Due to space limitations, we have skipped the proofs for this section. Please refer to [17] for details.

1) Across Rows: Let $r_{i}$ be the total number of $0 s$ across row $i$ in $P$, i.e. $r_{i}=\sum_{j=1}^{M}\left(1-p_{i j}\right)$, the minimum value of $k_{0}$ is given by

$$
k_{0} \geq \max _{i \in[1, N]}\left\{\left\lceil\frac{r_{i}}{q_{i}}\right\rceil\right\} .
$$

2) Along Columns: Let $p_{\max }$ be the maximum value among the upload limit vector $p$, i.e. $p_{\max }=\max _{i \in[1, N]}\left\{p_{i}\right\}$. Let $c_{j}$ be the total number of $1 s$ along column $j$ in $P$, i.e. $c_{j}=\sum_{i=1}^{N} P_{i j}$, we can find the minimum number of $1 s$ along all columns $c_{\min }=\min _{j \in[1, M]}\left\{c_{j}\right\}$. The minimum value of $k_{0}$ is given by

$$
k_{0} \geq\left\lceil\log _{1+P_{\max }} \frac{N}{c_{\min }}\right\rceil \text {. }
$$

3) Whole Matrix: Let $z$ be the total number of $0 s$ in $P$, i.e. $z=\sum_{i=1}^{N} \sum_{j=1}^{M}\left(1-P_{i j}\right)$. Let $p_{\text {sum }}$ be the sum of $p_{i}$ in $p$, i.e. $p_{\text {sum }}=\sum_{i=1}^{N} p_{i}$, and $q_{\text {sum }}$ be the sum of $q_{i}$ in $q$, i.e. $q_{\text {sum }}=\sum_{i=1}^{N} q_{i}$. The minimum value of $k_{0}$ is given by

$$
k_{0} \geq\left\lceil\frac{z}{\min \left\{p_{\text {sum }}, q_{\text {sum }}\right\}}\right\rceil .
$$

4) Lower Bound: Combining (2), (3), (4), the lower bound of the value of $k_{0}$ is the maximum value of the three, as in Eq. 5 (top of next page).

From simulations, we find (5) can return the optimal number of cycles for most cases we tested and we shall use the value estimated by (5) for performance comparison in later sections.

\section{SCheduling Algorithms}

We now present three types of transmission scheduling algorithms. They are Rarest Piece First (RPF), Most Demanding Node First (MDNF), and Maximum-Flow (MaxFlow) algorithms. All of them run in polynomial time.

\section{A. Rarest Piece First (RPF)}

The Rarest Piece First algorithm is borrowed from the Rarest Element First algorithm used in BitTorrent. In RPF, those file pieces that most peers do not have (rarest) are distributed first.

Definition 1: The rarity $c_{j}$ of Piece $j$ is the number of peers who have Piece $j$. That is, $c_{j}=\sum_{i=1}^{N} P_{i j}$.

$R P F$ aims at increasing the availability of different file pieces in the network, to maximize the chance that peers may still have some pieces that other peers want. In case the file is published by a single source who may just seed (remain available to contribute) the file for a short period of time, $R P F$ also tries to distribute all pieces from the original source to different peers across the network as quickly as possible, so that the distribution can continue even if the original source leaves.

In $R P F$, each peer chooses the rarest piece (with smallest $c_{j}$ ) to send out among those pieces he currently has. There is no preference on the choice of recipients; this piece is sent 


$$
k_{0} \geq \max \left\{\max _{i \in[1, N]}\left\{\left\lceil\frac{r_{i}}{q_{i}}\right\rceil\right\},\left\lceil\log _{1+P_{\max }} \frac{N}{c_{\min }}\right\rceil,\left\lceil\frac{z}{\min \left\{p_{\text {sum }}, q_{\text {sum }}\right\}}\right\rceil\right\} .
$$

to those with lower row indices who do not have this piece, have not been assigned to receive this piece from others and have not exceeded their download limits. If after distributing this piece to all possible recipients, the peer still has remaining upload bandwidth, he will try to distribute the next rarest piece until he has no more upload bandwidth or there are no more possible recipients. This process is done node by node (i.e. row by row in the possession matrix). For each cycle, the complexity of this algorithm is $O(N M(N+\log M))$.

$$
\mathrm{P}^{0}=\left(\begin{array}{llllllll}
1 & 0 & 1 & 0 & 0 & 0 & 0 & 0 \\
1 & 0 & 0 & 1 & 0 & 0 & 0 & 0 \\
0 & 1 & 0 & 0 & 1 & 0 & 1 & 1 \\
1 & 0 & 1 & 0 & 0 & 1 & 0 & 1 \\
0 & 1 & 0 & 0 & 0 & 0 & 1 & 1
\end{array}\right) P^{1}=\left(\begin{array}{llllllll}
1 & 0 & 1 & 1 & 1 & 0 & 0 & 0 \\
1 & 0 & 1 & 1 & 1 & 1 & 0 & 0 \\
0 & 1 & 0 & 0 & 1 & 1 & 1 & 1 \\
1 & 1 & 1 & 0 & 0 & 1 & 1 & 1 \\
0 & 1 & 0 & 0 & 0 & 0 & 1 & 1
\end{array}\right)
$$

For example, with $p=\{1,1,2,2,2\}$ and $q=\{2,3,2,3,3\}$, the schedule determined for $P^{0}$ above is (arrows are added for ease of understanding, and the resulting problem matrix at the next cycle, $P^{1}$, is also shown with those $1 s$ corresponding to the pieces just transmitted underlined.):

Node 1: send Piece 3 to Node 2

Node 2: send Piece 4 to Node 1

Node 3: send Piece 5 to Node 1, send Piece 5 to Node 2 Node 4: send Piece 6 to Node 2, send Piece 6 to Node 3 Node 5: send Piece 2 to Node 4, send Piece 7 to Node 4

However, we find the performance of $R P F$ is unsatisfactory and it performs much worse than the following algorithms.

\section{B. Most Demanding Node First (MDNF)}

As indicated in Section III.B, the number of cycles needed depends on two factors: how many pieces a peer needs and how rare a file piece is. $R P F$ only considers the second factor, while the Most Demanding Node First algorithm takes care of the first factor by adding one additional criterion for choosing recipients and the performance improvement over $R P F$ is significant with this simple enhancement.

Definition 2: The demand $d_{i}$ of Node $i$ is the number of un-received pieces for Node $i$. That is, $d_{i}=\sum_{j=1}^{M}\left(1-P_{i j}\right.$.

We attach a demand $d_{i}$ to every node and we prefer to send to the node with largest $d_{i}$. In case several nodes have the same demand, we just send to the node with the lowest row index. Similar to $R P F$, we schedule the transmissions node by node; while choosing recipients we prefer the one with the highest demand $d_{i}$ that does not have the piece and has not exceeded its download limit yet. Its complexity is also $O(N M(N+\log M))$. For example, with $p=\{1,1,2,2,2\}$ and $q=\{2,3,2,3,3\}$, the schedule is determined in this sequence:

Node 1: send Piece 3 to Node 2

Node 2: send Piece 4 to Node 1

Node 3: send Piece 5 to Node 1, send Piece 5 to Node 2

Node 4: send Piece 6 to Node 2, send Piece 6 to Node 5

Node 5: send Piece 2 to Node 4, send Piece 7 to Node 4

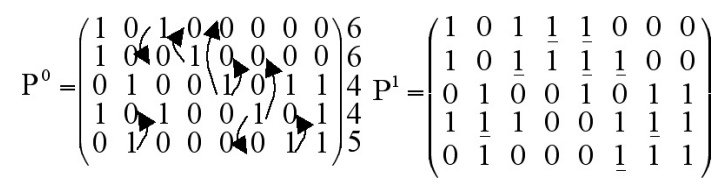

The demands for each node are written at the right of $P^{0}$. At $P^{0}$, Node 1 chooses its rarest piece, Piece 3, to send out and chooses the most demanding node, Node 2, to receive this piece. Node 4 now sends Piece 6 to Nodes 2 and 5 (instead of Node 3) because Node 5 is more demanding than Node 3.

$M D N F$ performs better than $R P F$ in most cases but is still not the best. A common characteristic that is shared between $R P F$ and $M D N F$ is that the maximum number of transmissions for each cycle cannot be achieved. In the following simple example, $p=\{2,2,1,1\}$ and $q=\{2,2,2,2\}$. Using MDNF, only five transmissions can be scheduled (but the maximum is six).

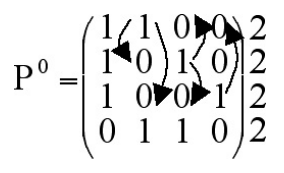

To fix the problem, we find as many transmissions as possible in each cycle and the algorithm is described in the next section.

\section{Maximum-Flow Algorithms (MaxFlow)}

In this section, we present a novel maximum-flow graph model for finding transmission schedules which outperforms the above two algorithms. We transform the problem instance to the well-known maximum-flow problem so as to find the maximum number of transmissions in each cycle. Weights are added to the nodes to achieve better matching. We first describe how to transform the problem and then explain the algorithm in detail.

1) Flow Network: A flow network graph $G=(V, E)$ is a directed graph in which each edge $(u, \nu) \in E$ has a nonnegative capacity $c(u, \nu) \geq 0$. There are two special vertices, the source $s$ and the sink $t$. A flow $f$ in $G$ is a real-valued function $f: V \times V \rightarrow R$ that satisfies the following three properties:

- Capacity Constraint: the flow from one vertex to another must not exceed the given capacity, i.e. $f(u, \nu) \leq c(u, \nu)$ $\forall u, \nu \in V$.

- Skew Symmetry: the flow from a vertex $u$ to a vertex $\nu$ is the negative of the flow in the reverse direction, i.e. $f(u, \nu)=-f(\nu, u) \forall u, \nu \in V$.

- Flow Conservation: the total flow out of a vertex other than the source or sink is zero, i.e. $\sum_{u \in V} f(u, \nu)=0$ $\forall \nu \in V-\{s, t\}$.

The value of a flow $f$ is defined as $|f|=\sum_{\nu \in V} f(s, \nu)$, i.e. the total flow out of the source. In the maximum-flow 


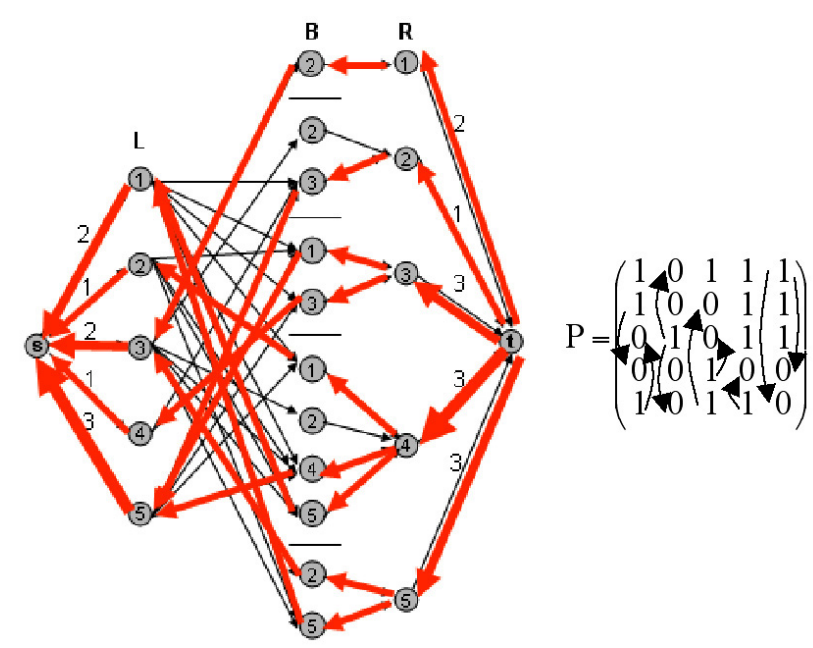

Fig. 2. Maximum-flow network graph and the scheduled transmissions.

problem, we wish to find a flow of maximum value. Due to space limitation, we refer interested readers to [16] for more formal discussions.

2) Problem Transformation: We now describe how to construct the flow network graph from a problem instance $P$.

Definition 3: The flow network graph from $P$ is a directed graph $G=(V, E)$. The vertices $V$ can be separated into four groups, i.e., $V=L \cup R \cup B \cup\{s, t\}$. $L=\left\{L_{1}, L_{2}, \ldots, L_{N}\right\}$ refers to the $N$ peers acting as senders in the file sharing session and $R=\left\{R_{1}, R_{2} \ldots, R_{N}\right\}$ refers to the same $N$ peers acting as receivers. $L$ and $R$ have the same cardinality as the number of peers $N$, i.e. $|L|=|R|=N$. For each node in $R$, we create an un-received piece node for each un-received file piece it demands and put these nodes into the set $B$, i.e. $b_{i j} \in$ $B \Leftrightarrow P_{i j}=0$. It has the same cardinality as the total number of $0 s$ in $P$, i.e. $|B|=\sum_{i=1}^{N} \sum_{j=1}^{M}\left(1-P_{i j}\right) . s, t$ are the source and sink nodes, respectively. The edges, $E=\left\{\left(s, L_{i}\right) \mid L_{i} \in L\right\} \cup$ $\left\{\left(R_{i}, t\right) \mid R_{i} \in R\right\} \cup\left\{\left(b_{i j}, R_{i}\right) \mid b_{i j} \in B, R_{i} \in R\right\} \cup H$, also consist of four sets. $\left\{\left(s, L_{i}\right) \mid L_{i} \in L\right\},\left\{\left(R_{i}, t\right) \mid R_{i} \in R\right\}$ are the sets of edges from the source $s$ to left-side nodes $L$ and right-side nodes $R$ to the sink $t$, respectively. $\left\{\left(b_{i j}, R_{i}\right) \mid b_{i j} \in B, R_{i} \in R\right\}$ are the edges from the unreceived piece nodes for node $i$ to the right-side node $R_{i} . H=$ $\left\{\left(L_{u}, b_{\nu j}\right) \mid L_{u} \in L, b_{\nu j} \in B\right.$, and $\left.\left(P_{u j}=1 \wedge P_{\nu j}=0\right)\right\}$ are the edges from left-side nodes $L$ to the un-received piece nodes $B$ and depend on the possession matrix $P$. There is an edge from $L_{u}$ to $b_{v j}$ if Peer $u$ has file piece $j$, while Peer $\nu$ does not have it, so that Peer $u$ can send Piece $j$ to Peer $\nu$. The edge capacities are integer-valued, $c\left(s, L_{i}\right)=p_{i}$ and $c\left(R_{i}, t\right)=q_{i}$, where $p_{i}$ and $q_{i}$ are the upload and download limits for Peer $i$ defined by the vectors $p=\left\{p_{1}, p_{2}, \ldots p_{N}\right\}$ and $q=\left\{q_{1}, q_{2}, \ldots q_{N}\right\} . c\left(s, L_{i}\right)$ dictates that Node $i$ sends at most $p_{i}$ pieces out. Similarly $c\left(R_{i}, t\right)$ dictates that Node $i$ receives at most $q_{i}$ pieces from others. $c(u, \nu)=1 \forall(u, \nu) \in$ $E \backslash\left(\left\{\left(s, L_{i}\right)\right\} \cup\left\{\left(R_{i}, t\right)\right\}\right)$ for other edges excluding those from source $s$ to $L_{i}$ and those from $R_{i}$ to sink $t$.

Fig. 2 illustrates the transformation process. For this possession matrix $P$, with $p=\{2,1,2,1,3\}$ and $q=\{2,1,3,3,3\}$, the flow network is shown beside $P$. There are a total of eleven un-received piece nodes in $B$, as there are eleven $0 s$ in $P$. $b_{12}$ corresponds to the un-received Piece 2 for Peer $1, b_{22}$ corresponds to Piece 2 for Node 2, etc. There are links from $L_{1}$ to $b_{23}, b_{31}, b_{44}, b_{55}$, etc., but not to $b_{22}, b_{42}, b_{52}$, etc., since Peer 1 can send Piece 3 to Peer 2, Piece 1 to Peer 3, Piece 4 to Peer 4, Piece 5 to Peer 5, but cannot send Piece 2 to other peers as Peer 1 does not have Piece 2 . The arguments for other links in $H$ are similar. The capacities for the edges from $s$ to $L_{i}$ and $R_{i}$ to $t$ are written on top of the corresponding edges and follow the given upload and download limit vectors $p$ and $q$. There are $O\left(N^{2} M\right)$ edges and the complexity for constructing the flow network graph from $P$ is also $O\left(N^{2} M\right)$.

To find the maximum-flow $\left|f^{*}\right|$, we adopt the wellestablished Edmonds-Karp algorithm, which is a particular implementation of the general Ford-Fulkerson method [16]. It finds augmenting paths by using breath-first search from the source $s$ to the sink $t$. Nodes with smaller indices have higher preference in expanding states in breath-first search. Its complexity is $O\left(|E|\left|f^{*}\right|\right)=O\left(N^{2} M \times \min \left\{\sum_{i=1}^{N} p_{i}, \sum_{i=1}^{N} q_{i}\right\}\right)$, which is still linear in $M$ and $N$, and is worth the implementation effort. In fact, the small increase in scheduling complexity would not add much to the overall transmission time as the computation time is very small compared with the actual data transmission time of file pieces.

For the example problem instance in Fig. 2, we will have a maximum-flow $\left|f^{*}\right|=9$, with matched flows highlighted. An example translation of a matched flow to a schedule is: $s \rightarrow L_{1} \rightarrow B_{45} \rightarrow R_{4} \rightarrow t$ means that Node 1 sends Piece 5 to Node 4. The transmission schedule is thus:

Node 1: send Piece 5 to Node 4, send Piece 5 to Node 5

Node 2: send Piece 1 to Node 4

Node 3: send Piece 2 to Node 1 , send Piece 2 to Node 5

Node 4: send Piece 3 to Node 3

Node 5: send Piece 3 to Node 2, send Piece 1 to Node 3, send Piece 4 to Node 4

3) MaxFlow - Weighted: Although MaxFlow always returns a schedule with the maximum number of transmissions for each cycle, the performance is unsatisfactory, as it does not consider whether we can match more in subsequent cycles. It does not take care of the rarity of file pieces and the demands of nodes as in $R P F$ and $M D N F$. To find a better matching, we put weights on the nodes in $L$ and $B$, so as to give priorities to some nodes during the matching process. Definitions 4 and 5 define how to measure the rarity of the file pieces a peer possesses and the rarity of the file pieces a peer demands, respectively.

Definition 4: The rarity possession index $\gamma_{i}$ of Peer $i$ is the sum of number of $0 s$ in other peers for those pieces that Peer $i$ has. That is, $\gamma_{i}=\sum_{a=1}^{N} \sum_{b=1}^{M}\left(A_{a b}(i)\right)$ where $A_{a b}$ is an $N \times M$ matrix and $A_{a b}(i)=$ $\left\{\begin{array}{ccc}1 & \text { if } & (a \neq i) \wedge \\ 0 & \text { if } & \left(P_{i b}=1\right) \wedge\left(P_{a b}=0\right) \\ \text { otherwise }\end{array}\right.$.

Definition 5: The rarity demand index $\delta_{i j}$ for file piece $j$ that Peer $i$ demands is the sum of number of $0 s$ across Row $i$ and Column $j$. That is, 

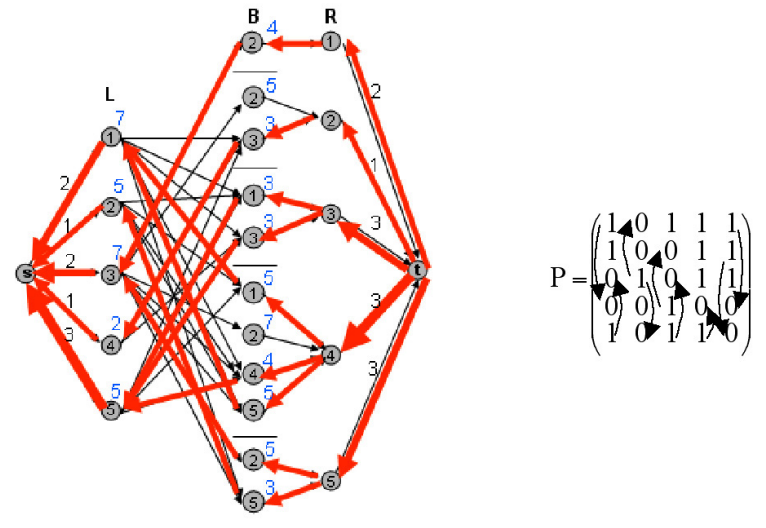

Fig. 3. MaxFlow - Weighted graph and its scheduled transmissions.

$$
\delta_{i j}=\sum_{g=1}^{M}\left(1-P_{i g}\right)+\sum_{h=1}^{N}\left(1-P_{h j}\right)-1 .
$$

Consider an example in Fig. 3, with $p=\{2,1,2,1,3\}$ and $q=\{2,1,3,3,3\}$. The numbers beside the nodes in $L$ are the rarity possession indices $\gamma_{i}$, and those beside the un-received piece nodes $B$ are the rarity demand indices $\delta_{i j}$. For example, $L_{3}$ has $\gamma_{3}=4+1+2=7$ because there are a total of seven $0 s$ along Columns 2, 4, and 5. $B_{42}$ has $\delta_{42}=7$ because there are a total of seven $0 s$ across Row 4 and along Column 2. By preferring paths with the largest $\gamma_{i}$ first, those peers who have rare pieces can send first; while by preferring paths with largest $\delta_{i j}$ first, those nodes with higher demand are selected to receive a rare piece first.

Simulation results show that MaxFlow - Weighted performs better than MDNF and RPF. However, there are still a few cases that it cannot achieve the optimal. The major reason is that the weights are kept constant for the whole duration of each cycle and will not be changed even if some partial assignments have been made. Thus, when choosing recipients those peers who have been assigned to receive something may still be further assigned to receive more as their static weights remain as the highest, resulting in unfair resource allocation.

4) MaxFlow - Dynamically-Weighted: Due to the above problem, we further enhance MaxFlow - Weighted by allowing the weights on nodes to be dynamically varied within each scheduling cycle. Whenever Peer $i$ is temporarily assigned to receive Piece $j$ during the scheduling process, the rarity possession indices $\gamma_{i}$ and rarity demand indices $\delta_{i j}$ will be changed and hence the weights on nodes will also be changed. Then the scheduling process continues using the new weights.

From simulations, MaxFlow - Dynamically-Weighted performs the best among all algorithms presented and it can achieve the optimal for most cases tested. It is the best algorithm we have developed so far.

\section{Simulation Results}

We randomly generate the problem instances (with each individual matrix element independently generated) and employ various algorithms presented above to find transmission schedules. We simulated 1000 cases for each setting. We note the average number of cycles needed for complete distribution

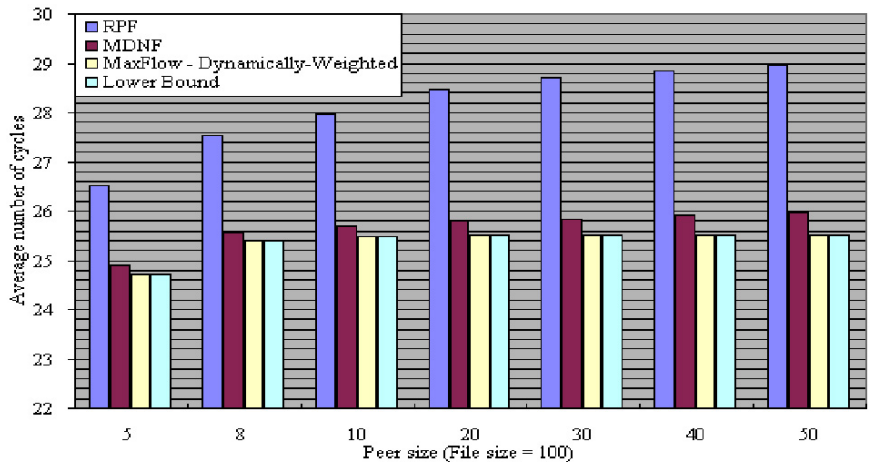

Fig. 4. Performance comparison of representative scheduling algorithms with varying peer sizes (file size $=100, p_{i}=2, q_{i}=3$, equal probability for $1 \mathrm{~s}$ and $0 \mathrm{~s})$.

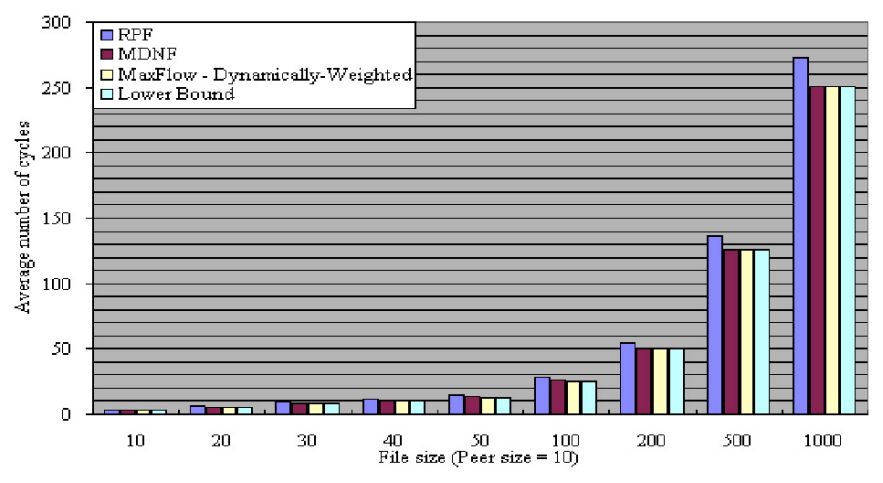

Fig. 5. Performance comparison of representative scheduling algorithms with varying file sizes (peer size $=10, p_{i}=2, q_{i}=3$, equal probability for $1 \mathrm{~s}$ and $0 \mathrm{~s})$.

by using that algorithm and compare it with the lower bound (5).

\section{A. Equal Probability of $1 s$ and $0 s$ in $P, p_{i}=2, q_{i}=3$}

For each matrix element in $P$, the probability of generating a 1 or 0 is the same, i.e. 0.5. All peers have the same upload and download limits of $p_{i}=2$ and $q_{i}=3$ for each cycle.

Fig. 4 and Fig. 5 show the performance comparison of the three representative algorithms with varying peer sizes and file sizes respectively. In all cases, the average number of cycles used by MaxFlow - Dynamically-Weighted is smaller than that by $M D N F$, which is in turn smaller than that by $R P F$. Also, MaxFlow-Dynamically-Weighted can achieve the same value as the lower bound in all cases, which implies that it already achieves the optimum for all cases tested in these graphs. For varying peer sizes as shown in Fig. 4, the average number of cycles used remains more or less constant with increasing peer size. This illustrates the good scalability of P2P file sharing approach; though more peers are requesting the same file, they also contribute their outgoing bandwidth for sharing. For varying file sizes as shown in Fig. 5, the performance improvement of MaxFlow - Dynamically-Weighted over RPF and $M D N F$ actually increases with increasing file size (though this cannot be easily seen due to the graph scale). In existing P2P file sharing networks, such as BT, the number of simultaneously connected peers is kept at about 10 30, while the number of file pieces is about $2000 \sim 4000$ for a typical 
file of 500MBytes 1GByte with file piece size of 256KBytes. The performance improvement of MaxFlow - DynamicallyWeighted over other algorithms becomes more significant with this large number of file pieces.

\section{B. Equal Probability of $1 s$ and $0 s$ in $P, p_{i}=3, q_{i}=7$}

We try different values of $p_{i}$ and $q_{i}$ to see if there is any significant effect on the performance. We try $p_{i}=3$ and $q_{i}=$ 7 , which is closer to the actual bandwidth allocation in ADSL. The results are similar to Fig. 4 and 5, demonstrating that variations in $p_{i}$ and $q_{i}$ will not have significant effects on the performance.

\section{Probability of $1 s: 0 s=1: 2, p_{i}=2, q_{i}=3$}

We try to have different probabilities for generating a 1 and a 0 for each matrix element in $P$, with the ratio of $1: 0$ to be $1: 2$, meaning that the network has fewer file pieces at the beginning, corresponding to an earlier stage of the file sharing session. Due to space limitation, please refer to the original graphs in [17].

When the network has fewer file pieces at the beginning, the performance improvement of MaxFlow - DynamicallyWeighted over RPF is greater than that when the network has more available file pieces. In this setting, when the peer size is 50 and the file size is 100, the percentage improvement of MaxFlow - Dynamically-Weighted over RPF is $21.28 \%$. For the same problem size in Fig. 4, the percentage improvement is only $13.56 \%$.

\section{DISCUSSION AND FUTURE WORK}

In this paper, we have investigated the simplified problem of P2P file distribution scheduling with asymmetric bandwidth allocation and synchronous scheduling assumptions. This scenario may appear in some private networks of a large company where some critical content has to be quickly replicated on a large number of machines. All the peers are known beforehand and they have similar connectivity and bandwidth capacities and the distribution process stops once the content has been fully replicated. Obviously, such static and synchronous scenarios are rare in real-world systems, but this preliminary study provides insights on the peer selection and file piece selection strategies, and evaluates their performance.

In our future research, we shall study the case of asynchronous scheduling in heterogeneous networks, in which the transmission time for sending a message between different pairs of nodes is different. We shall also study the case when the network is dynamic, in which the peers may come and go at will and they may shift to communicate with different sets of peers during the file distribution process.

\section{CONCLUSION}

Peer-to-Peer file-sharing applications have become immensely popular in the Internet, but previous research seldom investigates the data distribution problem which should be the core of any file sharing applications. We formally define the collaborative file distribution problem with the possession and transmission matrix formulation and deduce a theoretical bound for the minimum distribution time required. We develop several types of algorithms (RPF, MDNF and MaxFlow) for solving the scheduling problem of deciding who to send which file pieces, and to whom. In particular, our novel dynamically weighted maximum-flow algorithm outperforms other algorithms and can return the optimal solution for most cases as shown by simulations. Therefore, we conclude that the MaxFlow - Dynamically-Weighted algorithm is a promising algorithm for practical deployment in P2P file sharing applications.

\section{REFERENCES}

[1] The official BitTorrent website, http://www.bittorrent.com/

[2] The official Gnutella website, http://www.gnutella.com/

[3] The official Kazaa website, http://www.kazaa.com/

[4] The official Napster website, http://www.napster.com/

[5] A. Parker, "The true picture of peer-to-peer file-sharing," CacheLogic Presentation, July 2004.

[6] P. Rodriguez and E. W. Biersack, "Dynamic parallel access to replicated content in the Internet,"IEEE/ACM Trans. Networking, vol. 10, no. 4, pp. 455-465, Aug. 2002.

[7] X. Yang and G. de Veciana, "Service capacity of peer to peer networks," in Proc. IEEE INFOCOM, Mar. 2004.

[8] B. Cohen, "Incentive build robustness in BitTorrent," May 2003. [Online] Available: http://www.bittorrent.com/bittorrentecon.pdf

[9] E. W. Biersack, P. Rodriguez, and P. Felber, "Performance analysis of peer-to-peer networks for file distribution," in Proc. 5th Int'l Workshop on Quality of Future Internet Services, Sep. 2004, pp. 1-10.

[10] P. Felber and E. W. Biersack, "Cooperative content distribution: Scalability through self-organization," Self-star Properties in Complex Information Systems, O. Babaoglu et al., pp. 343-357. Berlin, Heidelberg: Springer-Verlag, 2005

[11] P. B. Bhat, C. S. Raghavendra, and V. K. Prasanna, "Efficient collective communication in distributed heterogeneous systems," in Proc. Int'l Conf. on Distributed Computing Systems, June 1999, pp. 15-24.

[12] S. Khuller and Y. A. Kim, "On broadcasting in heterogeneous networks," in Proc. ACM-SIAM Symposium on Discrete Algorithms, Jan. 2004

[13] T. S. E. Ng, Y. H. Chu, S. G. Rao, K. Sripanidkulchai, and H. Zhang, "Measurement-based optimization techniques for bandwidth-demanding peer-to-peer systems," in Proc. IEEE INFOCOM, Apr. 2003.

[14] D. Qiu and R. Srikant, "Modeling and performance analysis of BitTorrent-like peer-to-peer networks," in Proc. ACM SIGCOMM, Aug. 2004.

[15] J. S. K. Chan, V. O. K. Li, and K. S. Lui, "Scheduling algorithms for peer-to-peer collaborative file distribution," in Proc. Int'l Conf. on Collaborative Computing: Networking, Applications and Worksharing (CollaborateCom), Dec. 2005.

[16] T. H. Cormen, C. E. Leiserson, R. L. Rivest, and C. Stein, Introduction to Algorithms, 2nd ed. Cambridge, MA: MIT Press, 2001

[17] J. S. K. Chan, V. O. K. Li, and K. S. Lui, "Performance comparison of scheduling algorithms for peer-to-peer collaborative file distribution," Technical Report TR-2006-001, Department of Electrical and Electronic Engineering, University of Hong Kong, July 2006.

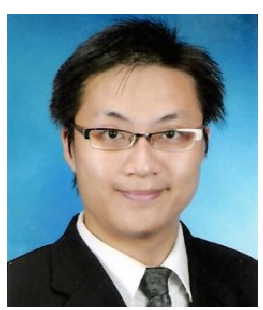

Jonathan S. K. Chan received the BEng degree (first class honour) in computer engineering from the University of Hong Kong (HKU), Pokfulam, Hong Kong in 2004. He is now working towards the MPhil degree in electrical and electronic engineering at $\mathrm{HKU}$.

His research interests include peer-to-peer networking, wireless and mobile computing, internet protocols and applications. 


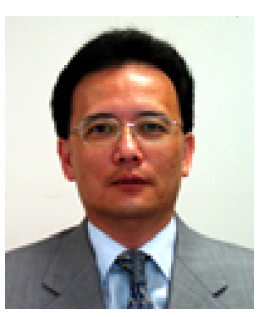

Victor O. K. Li received SB, SM, EE and ScD degrees in electrical engineering and computer science from the Massachusetts Institute of Technology, Cambridge, Massachusetts, in 1977, 1979, 1980, and 1981, respectively. He was elected an IEEE Fellow in 1992. He is also a Fellow of the HKIE and the IAE.

He joined the University of Southern California (USC), Los Angeles, CA, USA in February 1981, and became Professor of Electrical Engineering and Director of the USC Communication Sciences Institute. Since September 1997 he has been with the University of Hong Kong, where he is Chair Professor of Information Engineering at the Department of Electrical and Electronic Engineering. He has also been appointed Changjiang Chair Professor at Tsinghua University, Beijing, China. His research interests include all-optical networks, wireless networks, Internet technologies and applications.

Prof. Li has received numerous awards, including the UK Royal Academy of Engineering Senior Visiting Fellowship in Communications, the Croucher Foundation Senior Research Fellowship, and the Bronze Bauhinia Star, Government of the Hong Kong Special Administrative Region, China.

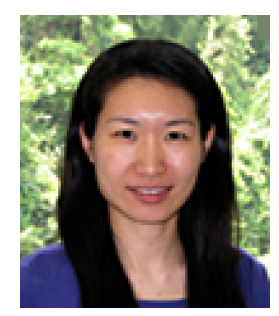

King-Shan Lui received the BEng (first class honor) and MPhil degrees in computer science from the Hong Kong University of Science and Technology, Hong Kong. She then received her PhD degree, also in computer science, from the University of Illinois at Urbana-Champaign in 2002.

She joined the Electrical and Electronic Engineering Department at the University of Hong Kong, Pokfulam, Hong Kong as an assistant professor in August 2002. Her research interests include network quality of service issues, topology aggregation, network protocol design and routing algorithm design. 